\title{
Infant Pulmonary Function Testing
}

\author{
Overview of Technology and Practical Considerations - \\ New Current Procedural Terminology Codes Effective 2010
}

\author{
Burton L. Lesnick, MD, FCCP; and Stephanie D. Davis, MD
}

\begin{abstract}
Infant pulmonary function testing has evolved from a research technique into a diagnostic tool. As such, new current procedural terminology (CPT) codes have been created and are available for use as of January 1, 2010. The technology now available has a range of applications. Through a series of vignettes, this article illustrates the methodology of the tests, some of their applications, and how to code and bill for the procedures.

CHEST 2011; 139(5):1197-1202

Abbreviations: $\mathrm{AMA}=$ American Medical Association; $\mathrm{CF}=$ cystic fibrosis; $\mathrm{CPT}=$ current procedural terminology; $\mathrm{ERV}=$ expiratory reserve volume; $\mathrm{FRC}=$ functional residual capacity; $\mathrm{RSV}=$ respiratory syncytial virus; $\mathrm{RUC}=\mathrm{Relative}$ Value Scale Update Committee; RV = residual volume; RVU = relative value unit
\end{abstract}

$\mathbf{W}$ hile children aged 5 years or older have been able to reliably perform pulmonary function tests via an active exhalation, it has been a challenge to assess the lung function of younger children. Tepper et $\mathrm{al}^{1}$ developed prototypes to measure infant pulmonary function in the 1980s that used an inflatable vest to squeeze the thoracic cage and measured passive airflow at the mouth. This technique was enhanced by using raised lung volumes in the mid-1990s., ${ }^{2,3}$ Normative data were subsequently developed and published. ${ }^{4,5}$ This method was further validated by a joint position statement from the American Thoracic Society and the European Respiratory Society in 2005. ${ }^{6}$ Over the following year, a six-part series of articles appeared in Pediatric Pulmonology that outlined the use of infant pulmonary function testing in evaluating chronic lung disease of infancy and acute neonatal respiratory disorders. ${ }^{7-12}$ The potential usefulness of this technique in defining disease has also been dem-

Manuscript received June 3, 2010; revision accepted September 26,2010

Affiliations: From Georgia Pediatric Pulmonary Associates (Dr Lesnick), Atlanta, GA; and Morehouse School of Medicine (Dr Lesnick), and the University of North Carolina at Chapel Hill (Dr Davis), Chapel Hill, NC.

Correspondence to: Burton L. Lesnick, MD, FCCP, 1100 Lake Hearn Dr, Ste 450, Atlanta, GA 30342; e-mail: blesnick@gppa.net (C) 2011 American College of Chest Physicians. Reproduction of this article is prohibited without written permission from the American College of Chest Physicians (http://www.chestpubs.org/ site/misc/reprints.xhtml).

DOI: 10.1378/chest.10-1423 onstrated in a number of publications. ${ }^{13-19}$ Devices have been approved recently by the US Food and Drug Administration and have been commercially marketed (nSpire Infant Pulmonary Laboratory; Longmont, Colorado; and Jaeger/Viasys Master Screen BabyBody; Yorba Linda, California), resulting in some degree of standardization of practice in the United States. In 2009, an application was made to the CPT Editorial Panel of the American Medical Association (AMA) for a set of current procedural terminology (CPT) codes for infant pulmonary function testing. These codes were subsequently evaluated by the Relative Value Scale Update Committee (RUC) of the AMA, based on the resource-based relative value scale. Through a series of vignettes, this article illustrates the clinical application of this new technology and how to correctly bill for the work performed. The relative value units (RVUs) are denoted.

\section{Methodology}

After consent is obtained, the physician sedates the infant. Typically, chloral hydrate is used, although some centers use benzodiazepines or propofol. The infant is fitted with an inflatable vest around the thoracic cage. When performing the partial-flow-volume maneuver (the original method used to produce flows), an airtight mask is placed over the infant's nose and mouth, and is connected to a computerized 
pneumograph. The pneumatic vest is rapidly inflated, compressing the thorax. The infant's functional residual capacity (FRC) is determined from tidal breathing loops, and a calculated maximum flow rate at the FRC is then generated after thoracic compression. This maximum flow at FRC is the main basis for comparison between subjects. A variety of additional techniques have been developed; the two most common are plethysmography, which measures FRC, and the raised-volume technique, which uses passive inflation of the infant's lungs to a specified volume prior to thoracic compression.

During plethysmography, an infant is placed in a body box or plethysmograph in a supine position with a face mask sealed around the nose and mouth with putty. To measure the FRC at end inspiration, the respiratory circuit is occluded at end inspiration for two to three cycles. The pressure inside the box is plotted against the pressure measured at the mouth, and the slopes of the inspiratory efforts are used to calculate the FRC. The procedure is repeated three to five times to ensure reproducibility.

During the raised-volume technique, a continuous flow of air of approximately 1.5 times the infant's measured peak inspiratory flow is directed via a T-piece circuit attached to a pneumotachometer. Typically, $30 \mathrm{~cm} \mathrm{H}_{2} \mathrm{O}$ pressure is applied to the nose and mouth via the face mask described above during consecutive spontaneous inspirations, followed by passive exhalation. These maneuvers are repeated until a short pause in respiration is noted at end expiration. This also helps to achieve chest wall relaxation. Then, another inflation maneuver is performed, using $30 \mathrm{~cm} \mathrm{H}_{2} \mathrm{O}$ pressure, followed by a forced expiration generated by rapidly pressurizing the pneumatic vest. This is performed at successively increasing vest pressures, $\leq 140 \mathrm{~cm} \mathrm{H}_{2} \mathrm{O}$ pressure, until no further increases in forced expiratory flows are observed. The key is achieving flow limitation. The jacket pressure required to demonstrate flow limitations is usually $10 \mathrm{~cm} \mathrm{H}_{2} \mathrm{O}$. The transmitted vest pressure is typically half of the pressure measured when inflating the vest. For the forced expiratory maneuver, the lung volume at $30 \mathrm{~cm} \mathrm{H}_{2} \mathrm{O}$ is written as V30. Residual volume ( $\left.\mathrm{RV}\right)$ is defined as the lung volume at the completion of the forced expiratory maneuver. FVC is the lung volume between V30 and the RV. Spirometric flows are then analyzed at $50 \%$ and $75 \%$ of the FVC, flows between $25 \%$ and $75 \%$, and the forced expiratory volume in $0.5 \mathrm{~s}$. Fractional lung volumes can also be calculated, including expiratory reserve volume (ERV). The ERV is derived from the raised-volume forced expiratory maneuver as the difference between the lung volume at the end of the maneuver (RV) and the point at which a stable end-expiratory level is again reached (FRC). The ERV is subtracted from the FRC as measured by the body plethysmograph to obtain the RV. The FVC is measured during the forced expiratory maneuver and added to the RV to obtain an estimate of the TLC.

For purposes of this article, we will focus on the measurements corresponding with the newly approved CPT codes. The following descriptive vignettes and work descriptions correspond closely to the AMA's CPT code book. All patients and physician names presented in the following vignettes are invented, and any resemblance to real persons is purely coincidental.

\section{Vignette 1}

Kaitlyn, a 5-month-old girl with cystic fibrosis (CF) is hospitalized with bronchiolitis caused by respiratory syncytial virus (RSV). Her parents are upset because, unlike her older sister with CF, she did not receive RSV prophylaxis because of recent changes in the guidelines issued by the American Academy of Pediatrics. On flexible bronchoscopy, a colony of Pseudomonas aeruginosa was found. Despite 2 weeks of IV antibiotics for the bacterial superinfection, she has a persistent cough. Her parents and care team are unsure whether there may be persistent airflow limitation from the CF pulmonary exacerbation. Because of this possibility, a third week of IV antibiotics is being considered. The cough may be related simply to the resolving RSV infection. The care team and family agree to have infant pulmonary function tests performed to determine the extent of any abnormalities in Kaitlyn's forced expiratory flows.

Because the pediatric pulmonologist on the $\mathrm{CF}$ care team is not trained in performing infant pulmonary function tests, Dr Julia Smith, a different pediatric pulmonologist, is asked to perform the procedure. Dr Smith is certified in Pediatric Advanced Life Support, as is required by her hospital for performing conscious sedation. Kaitlyn is kept without any oral feeds for several hours, per hospital protocol. Dr Smith reviews Kaitlyn's record, explains to her parents the procedure and its potential complications, including the risks of the moderate sedation. Dr Smith shows Kaitlyn's parents the equipment used, and she obtains written consent for both the procedure and the sedation.

A nurse administers chloral hydrate to Kaitlyn in a dark, quiet room while she is being monitored continuously for heart rate, respiratory rate, and pulse oximetry. Once Kaitlyn is asleep, a respiratory therapist helps Dr Smith place the pneumatic vest around her. Kaitlyn continues to be monitored with pulse oximetry, and the respiratory therapist watches closely throughout the procedure. A tight-fitting mask is placed over Kaitlyn's nose and mouth, attached to a pneumotachometer. On a computer monitor, Dr Smith is able to track flow/volume loops generated by Kaitlyn's tidal 
breathing. The low-volume point in these tracings is deemed to be the infant's FRC. Once this is determined, the computer assists in timing the inflation of the pneumatic vest around Kaitlyn's chest, just as she is nearing the end of an inhalation (although sometimes manual timing is needed). With the respiratory therapist's assistance, this is repeated three times, each time generating a partial-flow-volume loop. From time to time, the respiratory therapist makes sure that there is a tight seal over Kaitlyn's nose and mouth. The jacket pressure is increased until the flows no longer increase. The maximum flow at FRC is calculated and found to be consistent from trial to trial. Fortunately for Kaitlyn, Dr Smith finds that it is well within normal range for an infant of her length. Dr Smith also notes that the partial-flow-volume loop shows no concavity, suggesting that there is no limitation of the smallairways flow. The team also plans to obtain a raisedvolume-flow volume loop. Kaitlyn's lungs are passively inflated using the face mask, and the pneumatic vest is again inflated, this time at an elevated lung volume of $30 \mathrm{~cm} \mathrm{H}_{2} \mathrm{O}$ pressure. Unfortunately, Kaitlyn awakens with this maneuver. Dr Smith makes the clinical decision not to resedate her, deeming that there is sufficient information already. Kaitlyn is then observed and monitored by the nursing staff until completely awake, then permitted oral feeds.

Dr Smith evaluates the data to ensure accuracy, including review of the midmaximal flow rates, according to the American Thoracic Society/European Respiratory Society consensus statement. ${ }^{20}$ If Kaitlyn had previously had infant pulmonary function tests, Dr Smith would have made a comparison with available previous studies, looking for a significant interval change. She then makes a written report. Copies are placed with the child's hospital chart and sent to the primary care physician. Dr Smith discusses her findings with the CF care team. The decision is made to halt further antibiotic therapy and send Kaitlyn home the next day.

Dr Smith uses the following code to submit a charge for her services:

94011: Measurement of spirometric forced expiratory flows in an infant or child through 2 years of age ( 2.00 physician work RVUs).

The relatively high physician work value assigned by the RUC for this code reflects the extended physician time required to supervise this procedure. Also important is the relative intensity of the work, during which the infant's cartilaginous rib cage is squeezed to a point where very little RV may be present. Transient gas exchange abnormalities can result from this procedure, and rarely, the patient may need oxygen placed as the result of hypoxemia. Since conscious sedation is part of the procedure, as described by the CPT code, it is not billed separately. The primary physician caring for Kaitlyn in the hospital can still bill for a separate evaluation and treatment code for the day. Dr Smith has heard that a physician in a neighboring state has a sedation team doctor perform the infant pulmonary function test with a trained respiratory therapist. The pediatric pulmonologist interprets the study and submits the 94011 charge, while the sedation physician bills separately for the conscious sedation. She notes that this is not typically done since most pediatric pulmonologists prefer to be present to direct the care and to improve the quality of the data.

A variety of other applications exist for the measurement of spirometric forced expiratory flows. For instance, a child with interstitial lung disease might have diminished spirometric flows compared with normative data.

The RUC did not approve any practice expenses. This testing is done only in a hospital setting, so ambulatory patient classification outpatient hospital charges are used for the hospital to be reimbursed for its costs.

\section{Vignette 2}

Marcus is a 4-month-old boy, born at 28 weeks estimated gestational age. He was treated perinatally using continuous airway pressure for 2 weeks and only had transient placement of an endotracheal tube for exogenous surfactant administration in the delivery room. By 6 weeks of age (34 weeks postmenstrual), he was successfully weaned off supplemental oxygen. Unfortunately, he now has a chronic cough unrelated in timing to his feeds. His pediatrician orders infant pulmonary function testing to determine if there are abnormalities in forced expiratory flows and if they are reversible by using a bronchodilator.

Dr Smith reviews the child's history, writes a full report on the history and physical examination for the medical record, and discusses the procedure with Marcus' parents. She shows them the equipment and obtains consent for the sedation and the infant pulmonary function tests. After administration of the chloral hydrate, Marcus' nurse reports to Dr Smith that his oxygen saturation has dropped from a baseline of $96 \%$ to $93 \%$ during sleep. The decision is made to proceed with the testing. The care team continues as outlined above for Kaitlyn.

Marcus is placed in the airtight, clear box. After it is closed and a mask is adjusted over his nose and mouth, tidal breathing is measured. A set resistance is then applied to the circuit by closing a valve in the system. Marcus tidal breathes a minimum of three times against this set resistance. The maneuver is repeated 
two to three times. Using Boyle's law, the FRC is then calculated as described in the "Methodology" section. Next, a series of successive forced exhalations is performed. Unfortunately, Marcus' oxygen saturation drifts down to $88 \%$. Dr Smith then performs a passive inflation of Marcus' lungs to $30 \mathrm{~cm} \mathrm{H}_{2} \mathrm{O}$ pressure, noting an immediate improvement in saturation to $95 \%$. A series of raised-volume forced-exhalation maneuvers is performed as described in the "Methodology" section, concluding with another transient desaturation to 89\%. Dr Smith examines Marcus, noting scattered wheezes on the right side. This correlates with the concave flow-volume loops, suggesting an obstructive process. Marcus' ERV is calculated after a forced exhalation maneuver is performed using the pneumatic vest. Fractional lung volumes are calculated using these raised-volume measures and the FRC values. Two puffs of albuterol are administered via an in-line spacing chamber. After 15 min of waiting, Dr Smith reexamines Marcus and finds that his oxygen saturation has returned to $96 \%$ and his wheezes are absent. She makes the decision to return him to the infant lung function device for additional testing. This time, not only is there no drop in the oxygen saturation but also the flow-volume loops appear more normal in appearance.

Nonetheless, Dr Smith suggests overnight observation for Marcus. After analyzing the data as noted above, she communicates with the primary care physician and Marcus' parents. All involved agree to watch him overnight in the hospital. Dr Smith also recommends starting the use of inhaled budesonide routinely at home and follow-up care with a pediatric pulmonologist. Marcus does well and is discharged home by Dr Smith the next day. Out of habit, she documents 40 min of work on the discharge day, arranging for a home small-volume nebulizer and writing an asthma treatment plan for Marcus. Unlike the regular discharge after a full admission, the observation discharge codes are not time based.

Dr Smith lists her charges as follows:

94012: Measurement of spirometric forcedexpiratory flows, before and after bronchodilator use, in an infant or child through 2 years of age (3.10 physician work RVUs).

94013: Measurement of lung volumes (ie, FRC, FVC, and ERV) in an infant or child through 2 years of age ( 0.66 physician work RVUs). This is billed as two separate units because it was performed twice, once before the bronchodilator was used and once after, for a total of 1.32 physician work RVUs.

99219: Initial observation care, per day, for the evaluation and treatment of a patient, which requires these three key components: a compre- hensive history, a comprehensive examination, and medical decision making of moderate complexity. Counseling and/or coordination of care with other providers or agencies are provided consistent with the nature of the problems and the patient's and/or family's needs. Usually, the problems requiring admission for "observation status" are of moderate severity. (2.14 physician work RVUs).

And, on the day of discharge, the following code is submitted for billing:

99217: Observation care discharge-day treatment. (This code is to be used by the physician to report all services provided to a patient on discharge from "observation status" if the discharge is on other than the initial date of observation status. To report services for a patient designated as "observation status" or "inpatient status" and discharged on the same date, use the codes for Observation or Inpatient Care Services [including Admission and Discharge Services, 99234-99236, as appropriate.]) (1.28 physician work RVUs).

It should be noted that 94011 cannot be coded on the same day as 94012. ${ }^{21}$ The latter code encompasses the work performed in both the prebronchodilator and postbronchodilator studies.

\section{Vignette 3}

Chelsea is a 9-month-old girl who underwent resection of her right upper and right middle pulmonary lobes at 1 week of age. These areas had been affected by congenital lobar emphysema. Immediately postoperatively, her remaining lung tissue expanded, allowing normal gas exchange. She has since been growing well, but the family has kept her isolated from contact with any other young children. Her parents are wondering if her lungs have recovered sufficiently to allow her to attend daycare. Her pediatrician suggests assessing her lung volumes to determine compensatory growth.

Dr Smith meets the family in the day surgery area of the hospital, interviews them, reviews Chelsea's chest radiographs, and enters a report on Chelsea's history and physical exam into the chart. After showing the family the equipment and obtaining consent for the procedure and moderate sedation, Chelsea is sedated and positioned as described in the preceding vignettes. Using the airtight body plethysmograph, the FRC is measured. Following the performance of the plethysmographic technique, the pneumatic vest is used to measure forced expiratory flows at a raised lung volume. Chelsea's lungs are passively inflated to $30 \mathrm{~cm} \mathrm{H}_{2} \mathrm{O}$ pressure, and she then passively exhales. This inflation and passive exhalation is repeated until 
a short respiratory pause is noted. Next is the forced exhalation. After the lungs are passively inflated, the jacket around Chelsea's chest is rapidly inflated to produce flows at the raised lung volume. This maneuver is then repeated at increasing vest pressures until flow limitation is achieved. Calculations are made to determine the FVC and the forced expiratory volume in the first $0.5 \mathrm{~s}$.

Chelsea's FVC is found to be only $70 \%$ of what is predicted for her length. Dr Smith counsels the family about a mildly increased risk of pneumonia and suggests an annual influenza vaccination and consideration of the 23-valent pneumococcal vaccine at 24 months of age if follow-up testing continues to show mild restrictive lung disease. Last, the family is told to watch carefully for the development of scoliosis because of the unilateral nature of the loss in lung volume. Plans are made to repeat testing at 18 months. Dr Smith communicates the findings to the primary care physician and documents her report in the medical record. She submits the following charges:

94011: Measurement of spirometric forced expiratory flows in an infant or child through 2 years of age (2.00 physician work RVUs).

94013: Measurement of lung volumes (ie, FRC, FVC, and ERV) in an infant or child through 2 years of age ( 0.66 physician work RVUs).

Although the Collins equipment can provide measurements of respiratory system compliance and resistance, the current infant pulmonary function test CPT codes do not encompass this work. Code 94360 can be used to bill for airway resistance. Volume measurements (94240, Residual Lung Capacity, and 94260, Thoracic Gas Volume) are included in the infant pulmonary function test codes and should not be billed separately.

\section{SUMMARY}

Infant pulmonary function testing is now moving from a research tool to an addition to our clinical armamentarium. A variety of lung disorders in infants can be further assessed using this technique. Physicians are encouraged to familiarize themselves not only with the performance of these tests but also with the nuances of properly billing for them.

\section{ACKNOWLEDGMENTS}

Financial/nonfinancial disclosures: The authors have reported to CHEST the following conflicts of interest: Dr Davis receives grant support from the Cystic Fibrosis Foundation and the National Heart, Lung and Blood Institute. Dr Lesnick has reported to
CHEST that no potential conflicts of interest exist with any companies/organizations whose products or services may be discussed in this article.

\section{REFERENCES}

1. Tepper RS, Morgan WJ, Cota K, Wright A, Taussig LM. Physiologic growth and development of the lung during the first year of life. Am Rev Respir Dis. 1986;134(3):513-519.

2. Feher A, Castile R, Kisling J, et al. Flow limitation in normal infants: a new method for forced expiratory maneuvers from raised lung volumes. J Appl Physiol. 1996;80(6):2019-2025.

3. Turner DJ, Stick SM, Lesouëf KL, Sly PD, Lesouëf PN. A new technique to generate and assess forced expiration from raised lung volume in infants. Am J Respir Crit Care Med. 1995; 151(5):1441-1450.

4. Jones M, Castile R, Davis S, et al. Forced expiratory flows and volumes in infants. Normative data and lung growth. Am J Respir Crit Care Med. 2000;161(2 Pt 1):353-359.

5. Castile R, Filbrun D, Flucke R, Franklin W, McCoy K. Adulttype pulmonary function tests in infants without respiratory disease. Pediatr Pulmonol. 2000;30(3):215-227.

6. American Thoracic Society; European Respiratory Society. ATS/ERS statement: raised volume forced expirations in infants: guidelines for current practice. Am J Respir Crit Care Med. 2005;172(11):1463-1471.

7. Hülskamp G, Pillow JJ, Stocks J. Lung function testing in acute neonatal respiratory disorders and chronic lung disease of infancy: a review series. Pediatr Pulmonol. 2005;40(6): 467-470.

8. Hülskamp G, Pillow JJ, Dinger J, Stocks J. Lung function tests in neonates and infants with chronic lung disease of infancy: functional residual capacity. Pediatr Pulmonol. 2006;41(1): $1-22$.

9. Pillow JJ, Frerichs I, Stocks J. Lung function tests in neonates and infants with chronic lung disease: global and regional ventilation inhomogeneity. Pediatr Pulmonol. 2006;41(2): 105-121.

10. Lum S, Hülskamp G, Merkus P, Baraldi E, Hofhuis W, Stocks J. Lung function tests in neonates and infants with chronic lung disease: forced expiratory maneuvers. Pediatr Pulmonol. 2006;41(3):199-214.

11. Gappa M, Pillow JJ, Allen J, Mayer O, Stocks J. Lung function tests in neonates and infants with chronic lung disease: lung and chest-wall mechanics. Pediatr Pulmonol. 2006;41(4): 291-317.

12. Baldwin DN, Pillow JJ, Stocks J, Frey U. Lung-function tests in neonates and infants with chronic lung disease: tidal breathing and respiratory control. Pediatr Pulmonol. 2006;41(5):391-419.

13. Saito J, Harris WT, Gelfond J, et al. Physiologic, bronchoscopic, and bronchoalveolar lavage fluid findings in young children with recurrent wheeze and cough. Pediatr Pulmonol. 2006;41(8):709-719.

14. Sly PD. Objective assessment of lung disease in wheezy infants: the time has come. Pediatr Pulmonol. 2006;41(8): 798-800.

15. Brown DE, Pittman JE, Leigh MW, Fordham L, Davis SD. Early lung disease in young children with primary ciliary dyskinesia. Pediatr Pulmonol. 2008;43(5):514-516.

16. Tiddens HA, Donaldson SH, Rosenfeld M, Paré PD. Cystic fibrosis lung disease starts in the small airways: can we treat it more effectively? Pediatr Pulmonol. 2010;45(2):107-117.

17. Shell R, Allen E, Mutabagani K, et al. Compression of the trachea by the innominate artery in a 2 -month-old child. Pediatr Pulmonol. 2001;31(1):80-85. 
18. Linnane BM, Hall GL, Nolan G, et al; AREST-CF. Lung function in infants with cystic fibrosis diagnosed by newborn screening. Am J Respir Crit Care Med. 2008;178(12): 1238-1244.

19. Ranganathan SC, Stocks J, Dezateux C, et al. The evolution of airway function in early childhood following clinical diagnosis of cystic fibrosis. Am J Respir Crit Care Med. 2004; 169(8):928-933.
20. Sly PD, Tepper R, Henschen M, Gappa M, Stocks J; European Respiratory Society/American Thoracic Society. Tidal forced expirations. ERS/ATS Task Force on Standards for Infant Respiratory Function Testing. Eur Respir J. 2000;16(4):741-748.

21. Lesnick B, Mullholland M. Pediatric pulmonary and critical care coding. In: Manaker S, ed. Coding for Chest Medicine 2010. 14th ed. Northbrook, IL: American College of Chest Physicians; 2009:267-274. 